\title{
Genome Sequence Resource for Nigrospora oryzae, an Important Pathogenic Fungus Threatening Crop Production
}

\author{
Yafei Wang, ${ }^{1,2}$ Jinai Yao, ${ }^{3}$ Zhiqiang Li, ${ }^{2}$ Jianfei Huo, ${ }^{4}$ Shaoqun Zhou, ${ }^{1}$ Wende Liu, ${ }^{2, \dagger}$ and \\ Hanxiang $\mathrm{Wu}^{2, \dagger}$ \\ ${ }^{1}$ Shenzhen Branch, Guangdong Laboratory for Lingnan Modern Agriculture, Genome Analysis \\ Laboratory of the Ministry of Agriculture, Agricultural Genomics Institute at Shenzhen, Chinese \\ Academy of Agricultural Sciences, 440307, Shenzhen, China \\ ${ }^{2}$ State Key Laboratory for Biology of Plant Diseases and Insect Pests, Institute of Plant Protection, \\ Chinese Academy of Agricultural Sciences, 100193, Beijing, China \\ ${ }^{3}$ Fujian Key Laboratory for Monitoring and Integrated Management of Crop Pests/Institute of Plant \\ Protection, Fujian Academy of Agricultural Sciences, 350013, Fuzhou, China \\ ${ }^{4}$ Institute of Plant Protection, Tianjin Academy of Agricultural Sciences, 300381, Tianjin, China
}

\begin{abstract}
Nigrospora oryzae is an important phytopathogenic fungus with a broad host range. Here, we report an annotated draft of the genome of $N$. oryzae field strain GZL1 collected from maize assembled from PacBio and Illumina sequencing reads. The assembly we obtained has 15 scaffolds with an $N_{50}$ length of 4,037,616 bp. The resulting GZL1 draft genome is $43,214,190 \mathrm{bp}$, with GC content of $58.19 \%$. The completeness of GZL1 genome assembly is $99.30 \%$. This study is the first report of the genome sequence of $N$. oryzae, which can facilitate future study of the genetic variation and pathogenic mechanism of this important fungal pathogen.
\end{abstract}

\section{Genome Announcement}

Nigrospora oryzae is a globally distributed pathogenic fungus pathogen that is capable of infecting a wide variety of plants, including maize (Zea mays), rice (Oryza sativa), wheat (Triticum aestivum), sorghum (Sorghum bicolor), cotton (Gossypium spp.), and other crops (Fakhrunnisa et al. 2006; Hajano et al. 2011; Jagdish et al. 1985; Palmateer et al. 2003; Paul 1982; Prasad et al. 1960; Sharma et al. 2013; Standen 1945; Sun et al. 2021; Wu et al. 2014 Zhai et al. 2013; Zhang et al. 2012; Zheng et al. 2012; Zhong et al. 2016). The damaging impact of $N$. oryzae-caused diseases on agricultural production has been noted in the literature for over 70 years (Standen 1945). However, there has been no available information on the N. oryzae genome to date. The emergence of whole-genome sequencing technology and the subsequent application of the PacBio and lllumina strategy can generate genome assemblies for various important pathogenic fungi (Yun et al. 2019; Zhu et al. 2019). Here, we describe the first-draft genome assembly of $N$. oryzae, which will facilitate future studies of $N$. oryzae genomic features and the pathogenic mechanism of this fungus.

The field strain GZL1 was isolated from infected maize leaves from Gongzhuling, Jilin Province, China in October 2019. After single-spore purification, this strain was cultured on potato dextrose agar plates. Marginal hyphae were subcultured in potato dextrose broth at

${ }^{\dagger}$ Corresponding authors: W. Liu; liuwende@ caas.cn; and H. Wu; wuhanxiang@caas.cn

The author(s) declare no conflict of interest.

Accepted for publication 8 February 2021.

\section{Funding}

This work was financially supported by grants from the Agricultural Science and Technology Innovation Program (ASTIP) of the Chinese Academy of Agricultural Sciences.

\section{Keywords}

genomics, Nigrospora oryzae, PacBio sequencing, pathogenesis 
Table 1. Genome assembly statistics of Nigrospora oryzae field isolate GZL1

\begin{tabular}{lc} 
Variables & Statistics \\
Genome assembly size (bp) & $43,214,190$ \\
Number of scaffolds & 15 \\
Scaffold $N_{50}(\mathrm{bp})$ & $4,037,616$ \\
Scaffold $N_{90}(\mathrm{bp})$ & $1,823,678$ \\
Largest scaffold length (bp) & $10,312,485$ \\
GC content (\%) & 58.19 \\
BUSCO completeness (\%) & 99.3 \\
Number of genes & 10,039 \\
Number of virulence genes & 1,122 \\
Number of carbohydrate active enzymes & 478 \\
Number of secreted proteins & 1,037 \\
\hline
\end{tabular}

$25^{\circ} \mathrm{C}$ and $150 \mathrm{rpm}$ for 2 days. GZL1 was identified as N. oryzae based on morphological characteristics and internal transcribed spacer gene analysis (Gardes and Bruns 1993). The Omega Fungal DNA Kit D3390-02 was used to extract genomic DNA from fresh hyphae tissue following the manufacturer's instructions. DNA purity and content were evaluated with a TBS-380 fluorometer (Turner BioSystems Inc., Sunnyvale, CA, U.S.A.), and DNA integrity was tested with electrophoresis on a $0.8 \%$ agarose gel. The quality-controlled DNA sample was used for genome sequencing using a combination of PacBio Sequel Single-Molecule Real-Time (SMRT) and Illumina sequencing platforms. The sheared genomic DNA was used to prepare the SMRTbell library by SMRTbell sequencing adapters (Pacific Biosciences) following the manufacturer's recommendations, and an approximately 10-kb insert library was sequenced on one SMRT cell using standard methods at Shanghai Majorbio Bio-Pharm Technology Co., Ltd. After removing the low-quality and short subreads, Canu v1.7 software was used to assemble the remaining PacBio subreads. The Illumina sequencing library was prepared with the NEXTflex Rapid DNA-Seq Kit and used for 150-bp paired-end sequencing on the Illumina HiSeq 4000 platform. The Illumina data were used to polish the assembled genome by PacBio data with Pilon software (Walker et al. 2014). Putative gene models in the polished genome assembly were predicted with MAKER2 v2.31.9 (Cantarel et al. 2008), and annotated with Nonredundant Protein Database, Swiss-Prot, Pfam, Gene Ontology, Clusters of Orthologous Groups of Proteins, and Kyoto Encyclopedia of Genes and Genomes. The completeness of this draft genome sequence assembly was evaluated with BUSCO v3 (Waterhouse et al. 2018). Potential virulence genes were annotated with the Database of Virulence Factors (Lu et al. 2012).

PacBio SMRT sequencing and Illumina pair-end sequencing produced $7,680,385,597$ bp (sequencing depth $178 \times$ ) and 6,432,919,841 bp (sequencing depth 149x) of raw reads data, respectively. The assembled draft genome was 43,214,190 bp in size, and the GC content was $58.19 \%$. The size of the assembled genome is close to the revised genome size (43.41 Mb) obtained by evaluating the Illumina pair-end sequencing data using the Genomeye method. In total, 15 scaffolds were included, and the $N_{50}$ is 4,037,616 bp. It should be noted that four interval sequences from scaffold 15 (sequence ID: JADKYO010000015.1) showed high identities (85 to 95\%) to the mitochondrial genome of Xylaria hypoxylon strain SFY20170806 (sequence ID: NC_046734.1), indicating that this scaffold may be the mitochondrial genome. The completeness of GZL1 genome assembly was $99.30 \%$. Other features of the genome are shown in Table 1. In total, 10,039 genes were predicted. Studies have shown that carbohydrate active enzymes (CAZymes) are related to pathogenicity and play an important role in the interaction between pathogenic fungi and their hosts (O'Connell et al. 2012; van den Brink and de Vries 2011). In total, 478 CAZymes were putatively identified in the assembled draft genome, including 218 glycoside hydrolases, 114 auxiliary active family proteins, 76 carbohydrate esterases, 59 glycosyltransferases, 9 polysaccharide lyases, and 2 carbohydrate-binding modules. In total, 1,122 virulence genes related to pathogenesis defined by the DFVF motif were putatively annotated (Lu et al. 2012). In addition, 1,313 transmembrane proteins, 1,182 transport proteins and 1,037 secreted proteins were identified through searching in the TMHMM, TCDB, and SignalP databases (E-value cutoff $=1 \mathrm{e}-05)$, respectively (Krogh et al. 2001; Petersen et al. 2011; Saier et al. 2006). It is worth noting that 1,450 putative pathogen-host interacting 
protein-encoding genes were identified in the PHI database (E-value cutoff = 1e-05), 24 of which were identified to encode potential effectors. These genes may play an important role in the pathogenesis process of $N$. oryzae.

The repeat sequence database constructed with RepeatMasker v4.0.7 software was used to predict repeat sequences in the ZGL1 genome and the result showed that the repeat sequence ratio was $0.06 \%$. Transposable elements (TEs) often play an important role in the pathogenic variation of plant fungi (Faino et al. 2016; Raffaele and Kamoun 2012). We carried out ab initio TE prediction with RepeatMasker v4.0.7 software, and sequences similar to known repeated sequences were identified and classified in this study. Finally, we identified 296 TEs in the GZL1 genome, including 36 DNA transposons, 163 LINE retrotransposons, 86 SINE retrotransposons, and 11 LTR retrotransposons. This result was different from the previously reported transposon composition of Magnaporthe oryzae, where LTR retrotransposons were dominant (Bao et al. 2017). Previous reports showed that TEs could be a key contributor to genomic plasticity and virulence variation in plant-pathogenic fungi (Bao et al. 2017). Both $N$. oryzae and $M$. oryzae can cause serious diseases on rice but, unlike $M$. oryzae, the host range of $N$. oryzae is broad. The difference in the composition of transposons between $N$. oryzae and $M$. oryzae may be related to their pathogenicity and their host diversity. By analyzing the repeats and searching for telomere repeats on both ends of each assembled scaffold, telomeric repeats (CCCTAA) were identified on the $5^{\prime}$ ends of scaffolds 4 and 6 , and telomeric repeats (TTAGGG, which is the reverse complement of CCCTAA) were identified on the $3^{\prime}$ ends of scaffolds 3 and 7.

The GZL1 genome assembly described in this study is the first available genome of $N$. oryzae, which can enhance our understanding of the pathogenic mechanism of $N$. oryzae and the interaction between the pathogen and its host. The sequenced fungal isolate is stored in the State Key Laboratory for Biology of Plant Diseases and Insect Pests, Institute of Plant Protection, Chinese Academy of Agricultural Sciences, Beijing, China. The read data of PacBio and Illumina sequencing have been submitted to the NCBI Sequence Read Archive with accession number PRJNA673970. This Whole Genome Shotgun project has been deposited at DNA Data Bank of Japan/European Nucleotide Archive/GenBank under the accession JADKYO000000000. The version described in this article is version JADKYO010000000.

\section{Acknowledgments}

We thank J. Chen (Chinese Academy of Agricultural Sciences) for his continuous support and helpful discussions.

\section{Literature Cited}

Bao, J., Chen, M., Zhong, Z., Tang, W., Lin, L., Zhang, X., Jiang, H., Zhang, D., Miao, C., Tang, H., Zhang, J., Lu, G., Ming, R., Norvienyeku, J., Wang, B., and Wang, Z. 2017. PacBio sequencing reveals transposable elements as a key contributor to genomic plasticity and virulence variation in Magnaporthe oryzae. Mol. Plant 10:1465-1468.

Cantarel, B. L., Korf, I., Robb, S. M. C., Parra, G., Ross, E., Moore, B., Holt, C., Sánchez Alvarado, A., and Yandell, M. 2008. MAKER: An easy-to-use annotation pipeline designed for emerging model organism genomes. Genome Res. 18:188-196.

Faino, L., Seidl, M. F., Shi-Kunne, X., Pauper, M., van den Berg, G. C., Wittenberg, A. H., and Thomma, B. P. 2016. Transposons passively and actively contribute to evolution of the two-speed genome of a fungal pathogen. Genome Res. 26:1091-1100.

Fakhrunnisa, Hashmi, M. H., and Ghaffar, A. 2006. Seed-borne mycoflora of wheat, sorghum and barley. Pak. J. Bot. 38:185-192.

Gardes, M., and Bruns, T. D. 1993. ITS primers with enhanced specificity for basidiomycetes-Application to the identification of mycorrhizae and rusts. Mol. Ecol. 2:113-118.

Hajano, J.-u-d., Pathan, M. A., Rajput, Q. A., and Lodhi, M. A. 2011. Rice blast mycoflora, symptomatology and pathogenicity. Int. J. Agro Vet. Med. Sci. 5:53-63.
Jagdish, B. K., Laxminarayana, P., and Reddy, S. M. 1985. New records of post harvest diseases of oranges (Citrus reticulata). Indian Phytopathol. 38:575.

Krogh, A., Larsson, B., von Heijne, G., and Sonnhammer, E. L. L. 2001. Predicting transmembrane protein topology with a hidden Markov model: Application to complete genomes. J. Mol. Biol. 305:567-580.

Lu, T., Yao, B., and Zhang, C. 2012. DFVF: Database of fungal virulence factors. Database 2012:bas032.

O'Connell, R. J., Thon, M. R., Hacquard, S., Amyotte, S. G., Kleemann, J., Torres, M. F., Damm, U., Buiate, E. A., Epstein, L., Alkan, N., Altmüller, J., Alvarado-Balderrama, L., Bauser, C. A., Becker, C., Birren, B. W., Chen, Z., Choi, J., Crouch, J. A., Duvick, J. P., Farman, M. A., Gan, P., Heiman, D., Henrissat, B., Howard, R. J., Kabbage, M., Koch, C., Kracher, B., Kubo, Y., Law, A. D., Lebrun, M. H., Lee, Y. H., Miyara, I., Moore, N., Neumann, U., Nordström, K., Panaccione, D. G., Panstruga, R., Place, M., Proctor, R. H., Prusky, D., Rech, G., Reinhardt, R., Rollins, J. A., Rounsley, S., Schardl, C. L., Schwartz, D. C., Shenoy, N., Shirasu, K., Sikhakolli, U. R., Stüber, K., Sukno, S. A., Sweigard, J. A., Takano, Y., Takahara, H., Trail, F., van der Does, H. C., Voll, L. M., Will, I., Young, S., Zeng, Q., Zhang, J., Zhou, S., Dickman, M. B., Schulze-Lefert, P., Ver Loren van Themaat, E., Ma, L. J., and Vaillancourt, L. J. 2012. Lifestyle transitions in plant pathogenic Colletotrichum fungi deciphered by genome and transcriptome analyses. Nat. Genet. 44:1060-1065. 
Palmateer, A. J., McLean, K. S., van Santen, E., and Morgan-Jones, G. 2003. Occurrence of Nigrospora lint rot caused by Nigrospora oryzae on cotton in Alabama. Plant Dis. 87:873.

Paul, A. R. 1982. Winter leaf spot on borage caused by Nigrospora oryzae. Australas. Plant Pathol. 11:9-10.

Petersen, T. N., Brunak, S., von Heijne, G., and Nielsen, H. 2011. SignalP 4.0: Discriminating signal peptides from transmembrane regions. Nat. Methods 8:785-786.

Prasad, N., Agnihotri, J. P., and Agarwal, J. P. 1960. A new species of Nigrospora zinm on Oryza sativa L. Curr. Sci. 29:352-353.

Raffaele, S., and Kamoun, S. 2012. Genome evolution in filamentous plant pathogens: Why bigger can be better. Nat. Rev. Microbiol. 10:417-430.

Saier, M. H. J., Tran, C. V., and Barabote, R. D. 2006. TCDB: The Transporter Classification Database for membrane transport protein analyses and information. Nucleic Acids Res. 34:D181-D186.

Sharma, P., Meena, P. D., and Chauhan, J. S. 2013. First report of Nigrospora oryzae (Berk. \& Broome) Petch causing stem blight on Brassica juncea in India. J. Phytopathol. 161:439-441.

Standen, J. H. 1945. Nigrospora oryzae (B. and Br.) fetch on maize. Phytopathology 35:552-564.

Sun, X.-D., Cai, X.-L., Pang, Q.-Q., Zhou, M., Zhang, W., Chen, Y.-S., and Bian, Q. 2021. First record of leaf spot disease on Costus speciosus caused by Nigrospora oryzae in Hainan, China. Plant Dis. 105:506.

van den Brink, J., and de Vries, R. P. 2011. Fungal enzyme sets for plant polysaccharide degradation. Appl. Microbiol. Biotechnol. 91:1477-1492.

Walker, B. J., Abeel, T., Shea, T., Priest, M., Abouelliel, A., Sakthikumar, S., Cuomo, C. A., Zeng, Q., Wortman, J., Young, S. K., and Earl, A. M. 2014.
Pilon: An integrated tool for comprehensive microbial variant detection and genome assembly improvement. PLoS One 9:e112963.

Waterhouse, R. M., Seppey, M., Simão, F. A., Manni, M., Ioannidis, P. Klioutchnikov, G., Kriventseva, E. V., and Zdobnov, E. M. 2018. BUSCO applications from quality assessments to gene prediction and phylogenomics. Mol. Biol. Evol. 35:543-548.

Wu, J. B., Zhang, C. L., Mao, P. P., Qian, Y. S., and Wang, H. Z. 2014. First report of leaf spot caused by Nigrospora oryzae on Dendrobium candidum in China. Plant Dis. 98:996.

Yun, Y., Song, A., Bao, J., Chen, S., Lu, S., Cheng, C., Zheng, W., Wang, Z., and Zhang, L. 2019. Genome data of Fusarium oxysporum f. sp. cubense race 1 and tropical race 4 isolates using long-read sequencing. Mol. PlantMicrobe Interact. 32:1270-1272.

Zhai, L. F., Liu, J., Zhang, M. X., Hong, N., Wang, G. P., and Wang, L. P. 2013. The first report of leaf spots in Aloe vera caused by Nigrospora oryzae in China. Plant Dis. 97:1256.

Zhang, L. X., Li, S. S., Tan, G. J., Shen, J. T., and He, T. 2012. First report of Nigrospora oryzae causing leaf spot of cotton in China. Plant Dis. 96:1379.

Zheng, L., Shi, F., Kelly, D., and Hsiang, T. 2012. First report of leaf spot of Kentucky bluegrass (Poa pratensis) caused by Nigrospora oryzae in Ontario. Plant Dis. 96:909.

Zhong, J., Zhou, Q., Hu, Y., Zhu, H. J., and Da Gao, B. 2016. Molecular identification of a novel victorivirus from the phytopathogenic fungus Nigrospora oryzae. Virus Genes 52:156-159.

Zhu, B., Wang, S., Mi, C. Y., Yang, R. H., Zen, G. H., and Hu, X. F. 2019. Genome sequence resource for llyonectria mors-panacis, causing rusty root rot of Panax notoginseng. Mol. Plant-Microbe Interact. 32:1468-1471. 